\title{
Analysis of Destructive Effects with Electron Bombardment in Slow-Wave Structures
}

\author{
Nongchao Tan $\mathbb{D},{ }^{1,2,3}$ Ping Wu, ${ }^{2}$ Ye Hua, Jun Sun $\mathbb{D},{ }^{2}$ Yibing Cao, ${ }^{2}$ Guangshuai Zhang $\mathbb{D},{ }^{2}$ \\ Wenhui Huang $\mathbb{D},{ }^{1,3}$ and Wenhua Huang ${ }^{2}$ \\ ${ }^{1}$ Key Laboratory of Particle \& Radiation Imaging (Tsinghua University), Ministry of Education, Beijing 100084, China \\ ${ }^{2}$ Science and Technology on High Power Microwave Laboratory, Northwest Institute of Nuclear Technology, Xi'an, \\ Shaanxi 710024, China \\ ${ }^{3}$ Department of Engineering Physics, Tsinghua University, Beijing 100084, China
}

Correspondence should be addressed to Nongchao Tan; tnc17@mails.tsinghua.edu.cn

Received 2 September 2021; Revised 29 November 2021; Accepted 26 January 2022; Published 15 February 2022

Academic Editor: Katarzyna Batani

Copyright (C) 2022 Nongchao Tan et al. This is an open access article distributed under the Creative Commons Attribution License, which permits unrestricted use, distribution, and reproduction in any medium, provided the original work is properly cited.

Radio frequency (RF) breakdown can result in pulse shortening and seriously degrade the stability and reliability of relativistic backward wave oscillators (RBWOs). This paper discusses the energy range of electrons causing breakdown traces in slow-wave structures (SWSs) through particle-in-cell (PIC) simulation, numerical calculation, and experimental verification. The PIC simulation and numerical calculation results reveal that the energy of the majority of the field-induced electrons bombarding the SWS surfaces after being accelerated is less than $120 \mathrm{keV}$. Furthermore, the micro appearances of the breakdown traces in SWSs and the witness targets bombarded directly by electrons of various energy levels have been analyzed. Scanning electron microscope (SEM) shows that the breakdown traces are featured with corrugated morphologies with a wide range and a shallow depth. A mass of craters emerge in the vicinity of the corrugated morphologies. These appearances are quite similar to destructive traces impacted directly by low-energy electrons (around $160 \mathrm{keV}$ ). Thus, it is confirmed that the breakdown traces result from the bombardment of low-energy electrons. Therefore, the breakdown mechanism of field-emitted electrons impacting on the structure surfaces in RBWOs has been further improved.

\section{Introduction}

Relativistic backward wave oscillator (RBWO) is one of the most promising high-power microwave (HPM) devices owing to its compact structure, high-conversion efficiency, and repetitive operation [1-8]. In the past several decades, many methods have been proposed to continuously improve the power capacity and pulse width of RBWOs, such as the overmoded structures, the more effective resonant reflector, and the surface treating methods [9-14]. However, with the continuous increase of microwave output power, the intensity of the electromagnetic field on the structure surfaces increases accordingly, which will inevitably lead to the occurrence of radio frequency (RF) breakdown. The existence of RF breakdown causes irreversible structural damage, produces large amount of plasma, results in pulse shortening, and ultimately limits the performance of RBWOs [15-20]. The concept of explosive-electron emission (EEE) has been developed by many researchers to explain the vacuum breakdown in RBWOs [21, 22]. The associated physics includes the Coulomb explosion of microprotrusions at highly localized field-emitting sites, ionization of sputtered material, and the formation of cathode spot plasmas. However, it has been observed experimentally that the breakdown traces are more evident on the opposite sides of the locally strong field spots rather than the sides of the locally strong field, which is difficult to explain by EEE model [23]. Therefore, a few researchers have put forward that field-emitted electrons impacting on structure surfaces may be one of the causes of RF breakdown in RBWOs with a strong external guiding magnetic field [24]. When the strength of the electric field of a local region exceeds the 
field-emission threshold, field-induced electrons are generated. Afterwards, partial electrons can absorb energy from the RF field. Restricted by a strong guiding magnetic field, the energetic electrons bombard structure surfaces intensively and eventually cause material ablation and plasma formation. In our recent research, by observing the structure surfaces after different high-power microwave pulses, it has been verified experimentally that the breakdown traces on the structure surfaces originate from the bombardment of electrons [24]. However, the energy range of electrons bombarding the structure surfaces needs to be further studied, so as to provide theoretical guidance for proposing corresponding methods to suppress breakdown. In this paper, the formation mechanism of breakdown traces appearing in slow-wave structures (SWSs) after HPMgeneration experiment has been analyzed through simulation and experimental verification. By comparing the micro appearances of breakdown traces and witness plates bombarded by electrons of different energy levels, it is concluded that the energy of electrons causing structural damage is mainly less than $160 \mathrm{keV}$. This paper is organized as follows: in Section 2, field-emitted-electron movement in SWSs is studied using particle-in-cell (PIC) simulation. Section 3 presents single-particle calculation results, in close agreement with the PIC simulation results. Experimental verifications are demonstrated in Section 4. Finally, a brief summary and conclusion are given in Section 5.

\section{PIC Simulations}

Figure 1 shows a schematic diagram of an X-band RBWO, which consists of an annular cathode made of graphite, a resonant reflector, seven-period nonuniform SWSs, an extractor, and a beam collector. Under the restriction of an external strengthened magnetic field, an intensive relativistic electron beam (IREB) emitted by the annular cathode successively passes through the reflector, SWSs, and the extractor and ultimately is collected by the beam collector. During the process of IREB transmission, the electrons interact with the first spatial harmonic of the backward wave. The amplified backward wave is reflected by the reflector and becomes a forward wave afterwards. The extractor is mainly used to enhance the beam-microwave power conversion efficiency. Two-dimensional simulation models are carried out using the electromagnetic particle-in-cell (PIC) code UNIPIC, with a $0.25 \mathrm{~mm} \times 0.25 \mathrm{~mm}$ uniform grid [25]. With an $890 \mathrm{keV}, 12.6 \mathrm{kA}$ electron beam, the output power of the $\mathrm{X}$-band RBWO can reach $5.1 \mathrm{GW}$. The strongest normal electric field in the SWSs corresponding to the easiest emission of electrons is located in the left-hand side of the fourth high-frequency structure (about $620 \mathrm{kV} / \mathrm{cm}$ ).

The electron-emission boundary is set as the small radius of the high-frequency structure ridges, where breakdown traces first appear in the experiment. The beam emission model has been adopted in the PIC simulation. Fieldemitted-electron movement in SWSs can be monitored by real and phase spaces. SWS ridges can be divided into two types. The field-emitted electrons produced by several SWS ridges can bombard the opposite sides of the emitting sides

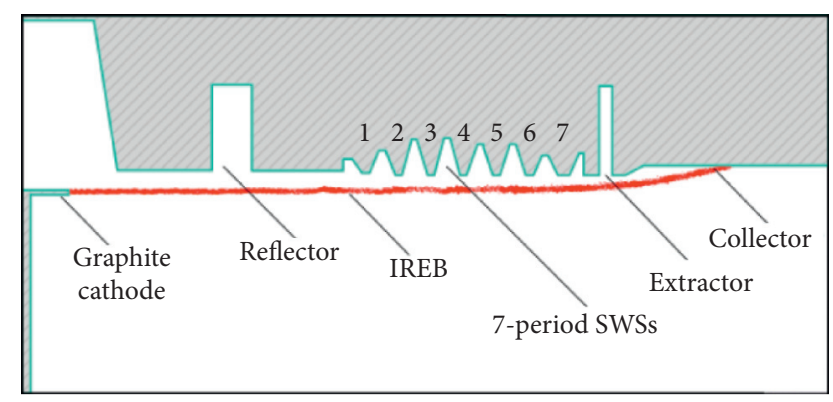

Figure 1: Schematic of the X-band RBWO.

after acceleration, as well as the emitting sides. The righthand side of the first high-frequency structure is given as an example, the real and phase space of the field-emitted electrons at which time $t(t=20 \mathrm{~s})$ is shown in Figure 2. The maximum electric field strength of the right-hand side of the first high-frequency structure is about $500 \mathrm{kV} / \mathrm{cm}$. According to Fowler-Nordheim formula, field-emission current depends on the locally applied electric field, equivalent field-enhancement factor $(\beta)$, and work function $\left(\varphi_{w}\right)$ [26]. In the previous study, $\beta$ ranges from tens to hundreds $[27,28]$. Setting $\beta=50, \varphi_{w}=4.3 \mathrm{eV}$, the corresponding current density is $1.36 \times 10^{8} \mathrm{~A} / \mathrm{m}^{2}$. Assuming that individual emission microdots are $10 \mu \mathrm{m}$ in size and 10 emission microdots exist in a single grid, the average current density of the grid can be calculated as $2.17 \times 10^{6} \mathrm{~A} / \mathrm{m}^{2}$. Furthermore, if the uniform distribution of the electric field along the angular direction is considered, the emission current intensity is $83 \mathrm{~A}$. Through the observation of momentum phase space (Figure 2(b)) and energy phase space (Figure 2(c)), the energy of the electrons that ultimately impacts on the opposite side and bombard back the emitting side is less than $100 \mathrm{keV}$. Besides, increasing or decreasing the emission current density to some extent has little influence on the emission electron motion. On the other hand, the field-emitted electrons generated by the other ridges of SWS are unable to transit the cavities between SWS rings to bombard the opposite sides; that is, they all return to the emitting sides. The real and phase space of the field-induced electrons at the left-hand side of the fourth high-frequency structure at time $t(t=20 \mathrm{~s})$ is shown in Figure 3. At this time, the maximum electric field strength of the left-hand side of the fourth high-frequency structure is $620 \mathrm{kV} / \mathrm{cm}$. Correspondingly, the average current density of the grid and the emission current intensity are $3.89 \times 10^{7} \mathrm{~A} / \mathrm{m}^{2}$ and $1.48 \mathrm{kA}$, respectively. Figure 3(c) shows that the energy of the electrons impacting back the emitting side is in the range of tens of $\mathrm{keV}$.

\section{Numerical Calculations}

To further analyze the energy distribution of the field-induced electrons impacting on SWS surfaces, single-particle calculations have been carried out.

Similar to the PIC simulation, the initial position of the electrons is set as the small radius of the SWS ridges. Although the field-enhancement factor $(\beta)$ is taken into 


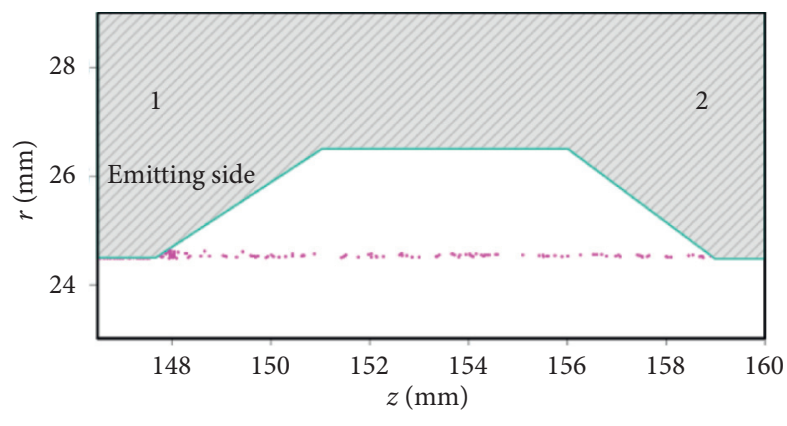

(a)

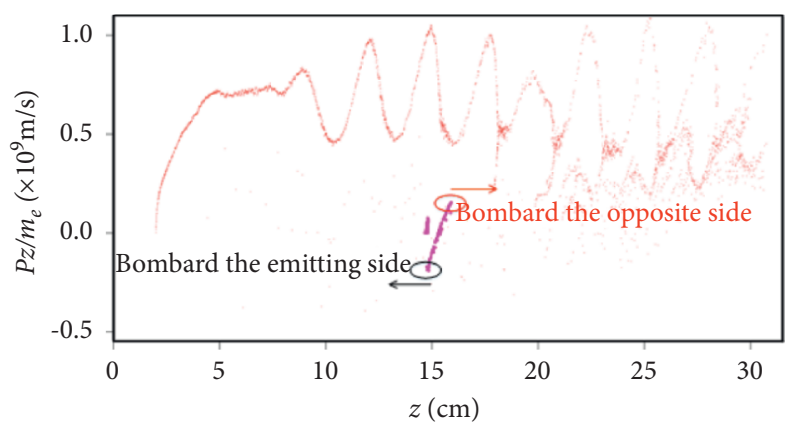

(b)

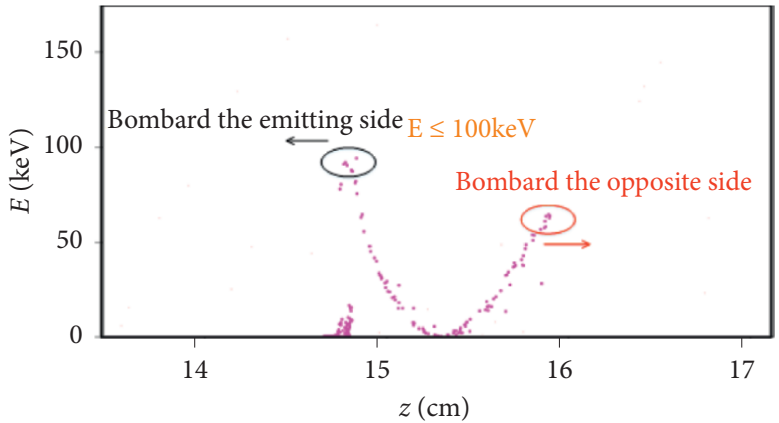

(c)

Figure 2: Movement of electrons produced at the right-hand side of the first high-frequency structure at time $t(t=20 \mathrm{~s})$. (a) Real space. (b) Momentum phase space. (c) Energy phase space.

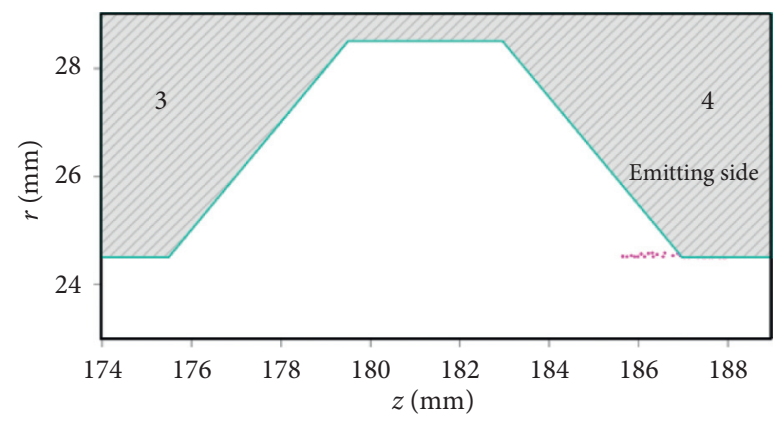

(a)

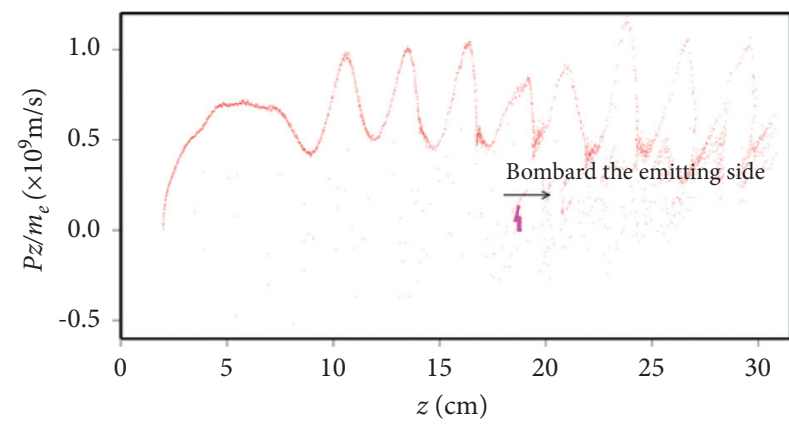

(b)

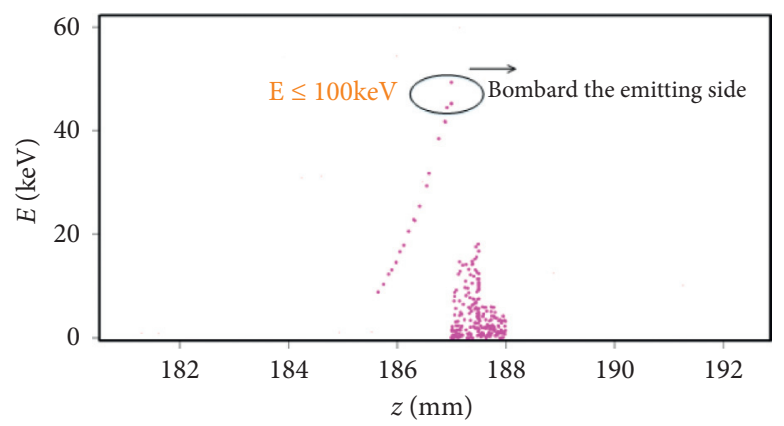

(c)

Figure 3: Movement of electrons produced at the left-hand side of the fourth high-frequency structure at time $t(t=20 \mathrm{~s})$. (a) Real space. (b) Momentum phase space. (c) Energy phase space.

consideration, the field-emission current of single microdot obtained using the Fowler-Nordheim formula is only a few amperes, and thus the space-charge field of this current can be negligible $[26,29]$. Therefore, the electrons are accelerated under the combined action of the RF electromagnetic field, the space-charge field induced by the IREB, and the external 
guiding magnetic field. The equation of motion can be written as

$$
\begin{aligned}
\frac{d}{d t}\left(\gamma m_{e} \dot{r}\right)-\gamma m_{e} r \dot{\theta} & =(-e) E_{r}+(-e)\left(r \dot{\theta}\left(B_{z}+B_{\text {out }}\right)-\dot{z} B_{\theta}\right), \\
\frac{1}{r} \frac{d\left(\gamma m_{e} r^{2} \dot{\theta}\right)}{d t} & =(-e)\left(\dot{z} B_{r}-\dot{r}\left(B_{z}+B_{\text {out }}\right)\right)+(-e) E_{\theta}, \\
\frac{d\left(\gamma m_{e} \dot{z}\right)}{d t} & =(-e) E_{z}+(-e)\left(\dot{r} B_{\theta}-r \dot{\theta} B_{r}\right),
\end{aligned}
$$

where $E_{z}, E_{r}, E_{\theta}, B_{z}, B_{r}$, and $B_{\theta}$ comprise the total field, the RF field combined with the space-charge field of the IREB extracted from the PIC simulation results; and $B_{\text {out }}$ suggests the external guiding magnetic field. The amplitude of the total electric field distribution in the SWSs at time $t(t=20 \mathrm{~s})$ is demonstrated in Figure 4. Due to the superposition of the space-charge field of the IREB, the field distribution is distorted in the position of the IREB.

The space gap $\Delta z$ and time interval $\Delta t$ of the extracted field are $0.25 \mathrm{~mm}$ and $T / 36$, respectively, where $\Delta z$ denotes the grid size and $T$ the corresponding RF period. The strength of the external magnetic field is selected as $4.3 \mathrm{~T}$. A time-centered differential leaping algorithm is adopted to maintain the propulsion of electron motion as follows:

$$
\frac{u^{t+\Delta t / 2}-u^{t-\Delta t / 2}}{\Delta t}=\frac{q}{m_{0}}\left(E^{t}+\frac{u^{t+\Delta t / 2}+u^{t-\Delta t / 2}}{2 \gamma^{t}} \times B^{t}\right)
$$

Similar to the PIC simulation (Section 2), the results of single-particle calculation can be divided into two categories as well. The movement of field-emitted electrons generated at the right-hand ridge of the first SWS is shown in Figure 5. Due to the superposition of the space-charge field, the emission time of electrons is less than half a microwave period. The field-emitted electrons at the right-hand ridge of the first SWS may be generated from the ninth time step to the nineteenth time step. Besides, only a proportion of the electrons emitted at a specific moment can transit through the cavity and bombard the opposite side, while most of the remaining electrons return to their emitting side. Specifically, most of the electrons generated at the ninth time step can transit through the cavity and bombard the opposite side (Figure 5(a)); in the next time step (the tenth time step), fewer electrons can bombard the opposite side (Figure 5(b)). All the electrons generated in other time steps can only return to their emitting side. The energy distribution of electrons bombarding the opposite side and returning to their own side is illustrated in Figure 6. Different colors represent the electrons generated from the ninth time step to the nineteenth time step. The energy of electrons bombarding both sides is mainly less than $120 \mathrm{keV}$. For the case where all generated electrons bombard their emitting side, the left-hand ridge of the fourth SWS is discussed as an example. During the emission period, the energy of the electrons that finally bombard back to their emitting side is mainly less than $100 \mathrm{keV}$, as shown in Figure 7.

The PIC simulation and single-particle calculation results reveal that the energy of the field-induced electrons bombarding the structure surfaces after being accelerated is

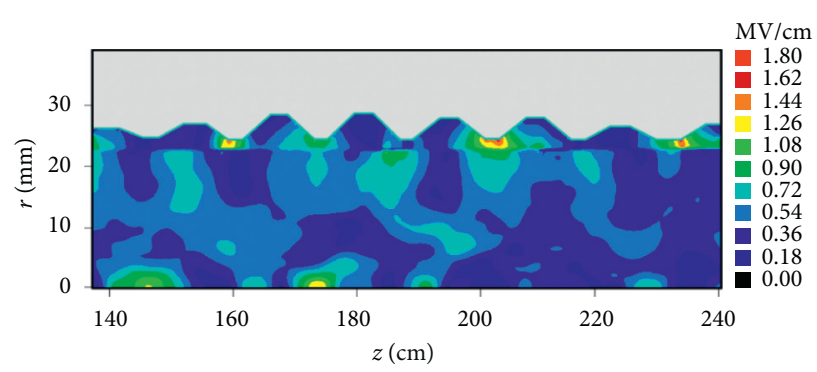

FIgURE 4: The amplitude of the total electric field distribution at time $t(t=20 \mathrm{~s})$.

mainly less than $120 \mathrm{keV}$. To verify this, the morphologies of the witness plates bombarded directly by the electron beam and the breakdown traces of SWSs after the HPM experiment are compared.

\section{Experimental Results}

An experiment involving an electron beam bombarding target plates made of stainless steel (SS) and titanium-alloy has been performed first. The morphologies of the SS witness plates bombarded by different energy electrons were obtained using a scanning electron microscope (SEM) and a three-dimensional confocal laser scanning microscope (CLSM), as shown in Figure 8. Compared that high-energy electrons (around $300 \mathrm{keV}$ ) are inclined to cause independent pit-like traces with smaller size and deeper depth (Figure 8(c)), the SS's surface tends to show corrugated morphologies with larger range but shallower depth after low-energy electron bombardment (around $160 \mathrm{keV}$ ) (Figure 8(a)). The lower the electron energy, the shallower and wider the damage traces. The three-dimensional morphologies of the damage traces imaged by the CLSM further verify the foregoing conclusion. The maximum depth of the damage traces is $23.5 \mu \mathrm{m}$ at $163 \mathrm{kV}$ and $48.7 \mu \mathrm{m}$ at $304 \mathrm{kV}$ (Figures $8(\mathrm{~d})$ and $8(\mathrm{e})$ ). Meanwhile, many craters emerge in the vicinity of corrugated morphologies after being impacted by low-energy electrons (Figure $8(\mathrm{~b})$ ). The formation of craters can be explained by the "melting mode" of the interaction between electrons and SS [30]. As electrons strike a material, the maximum energy deposition density is not located on the material's surface, but at a certain depth inside the material. During the interaction between the electrons and SS, part of the subsurface will reach the melting point first, resulting in the formation of molten droplets. Thereafter, under the quasistatic stress generated by the temperature field gradient, the droplets break through the surface to form eruptions, and near-circular holes are formed.

After interacting with electron beams, the surface of titanium-alloy witness plates shows destructive traces similar to that of SS, as depicted in Figure 9. The maximum depth of damage traces is $40.1 \mu \mathrm{m}$ at $166 \mathrm{kV}$ and $58.3 \mu \mathrm{m}$ at $303 \mathrm{kV}$ (Figures 9(c) and 9(d)), deeper than the traces formed on the surface of SS. This phenomenon can be explained by the energy deposition curve of electrons in materials. The density of SS is higher than that of titanium- 


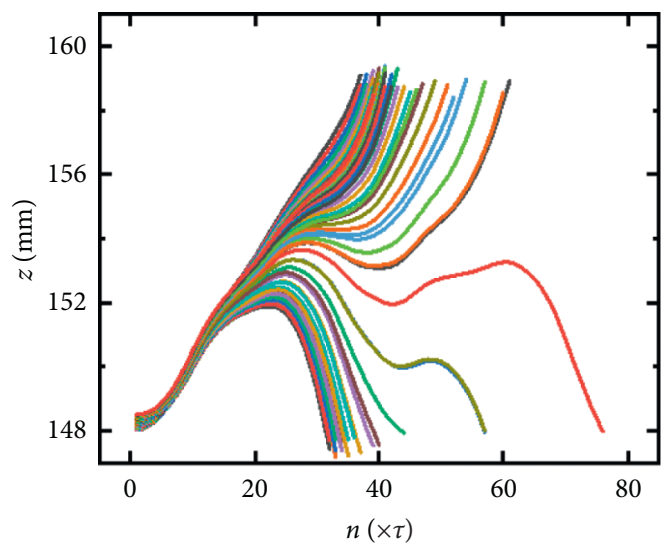

(a)

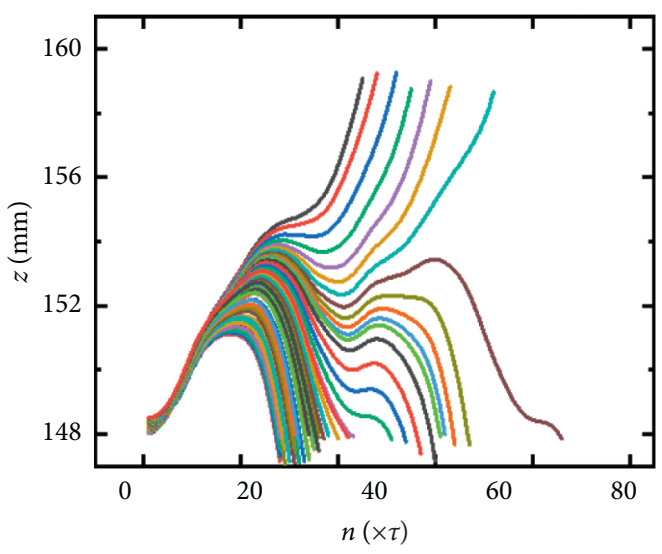

(b)

FIgURE 5: The axial position of the electrons generated at the ninth time step (a) and the tenth time step (b).

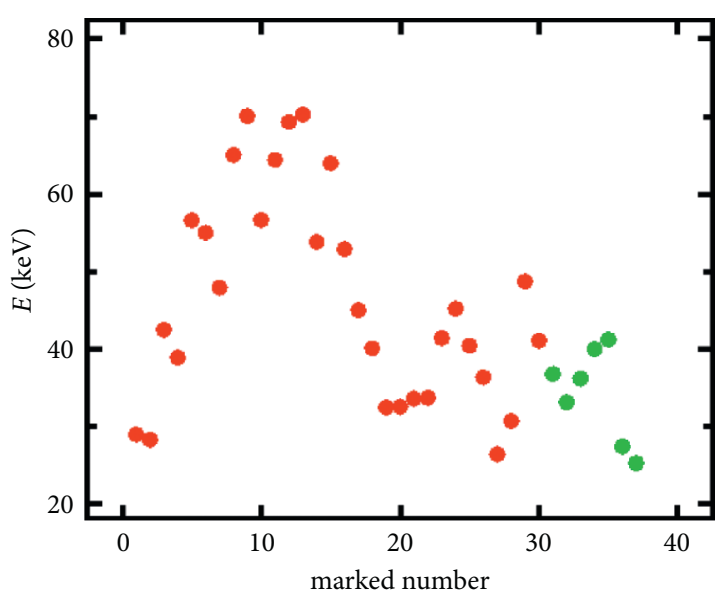

(a)

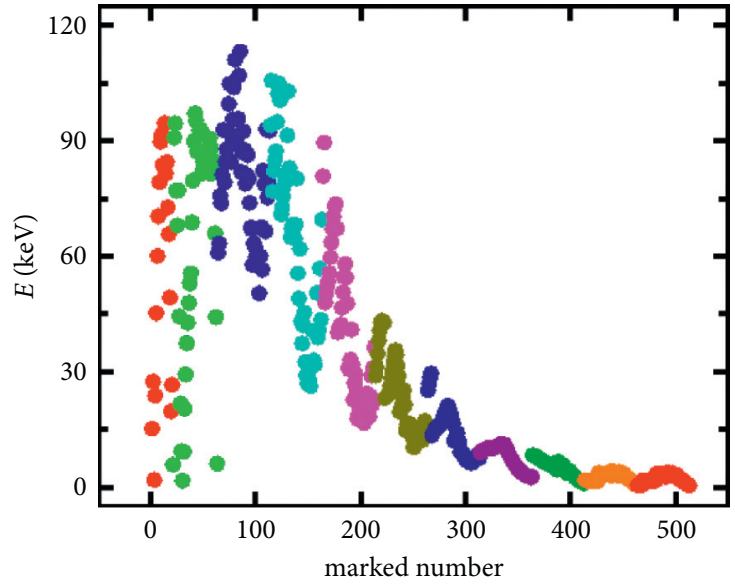

(b)

FIGURE 6: Energy distribution of electrons bombarding the opposite side (a) and the emitting side (b) (different colors indicate the electrons generated from the ninth time step to the nineteenth time step).

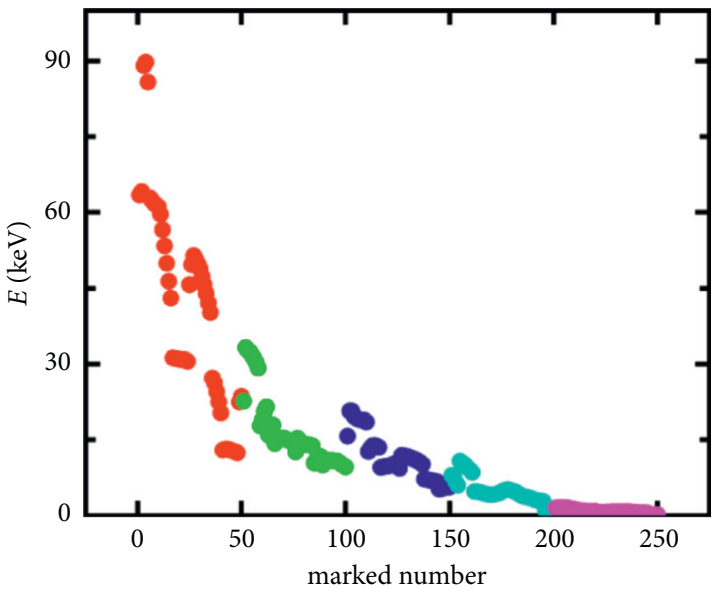

FIGURE 7: Energy distribution of electrons impacting on their emitting side only (different colors represent electrons emitted from the fourth time step to the eighth time step). 

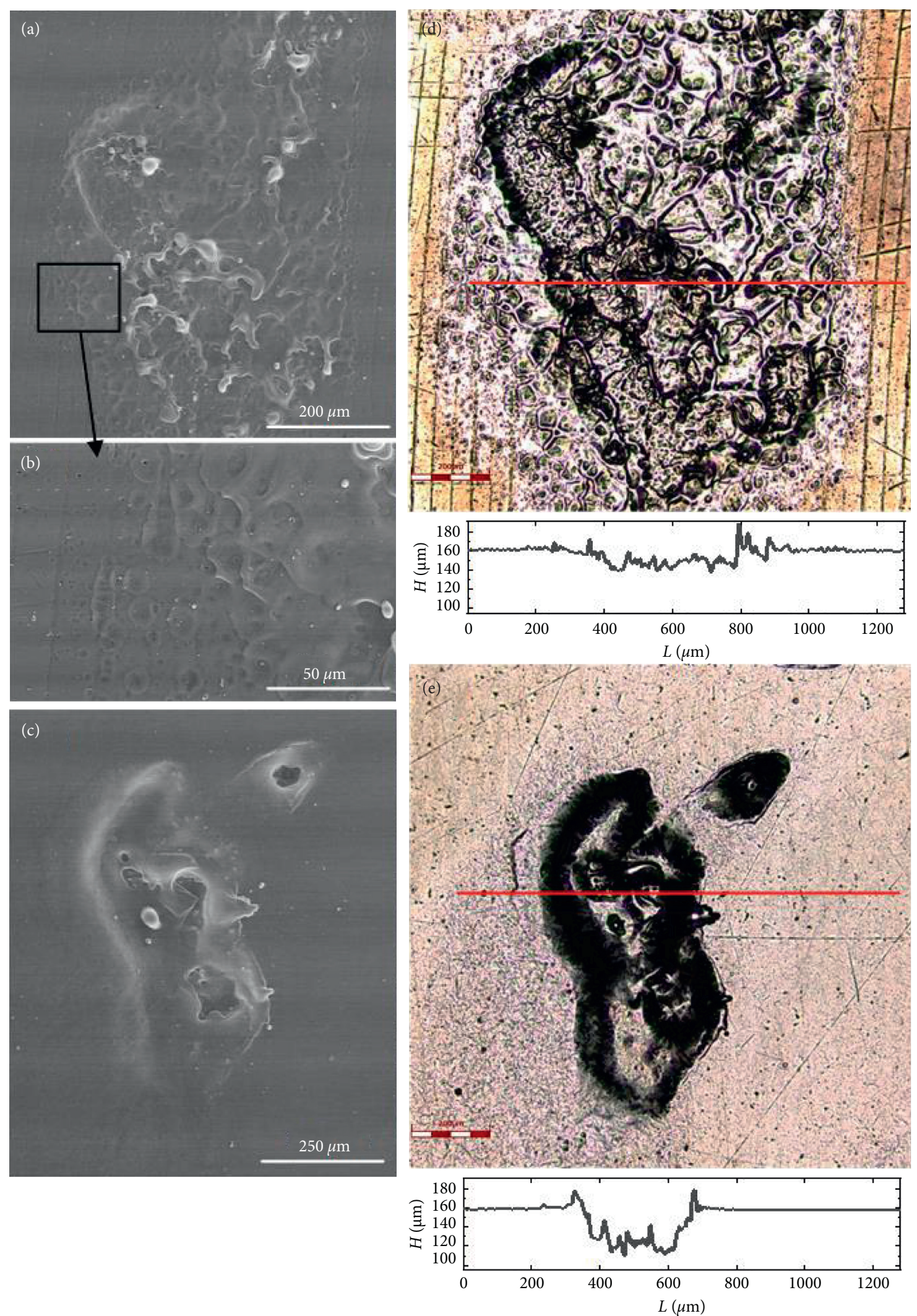

Figure 8: Microscopic images of damage traces on witness plates made of SS using a SEM (a-c) and a CLSM (d, e). (a) Corrugated morphologies appear at a diode voltage of $163 \mathrm{kV}$ and diode current of $1.59 \mathrm{kA}$. (b) Craters emerge in the vicinity of corrugated morphologies. (c) Independent, pit-like traces at a diode voltage of $304 \mathrm{kV}$ and diode current of $1.58 \mathrm{kA}$. (d) Three-dimensional morphologies of corrugated morphologies. (e) Three-dimensional morphologies of independent, pit-like traces. 


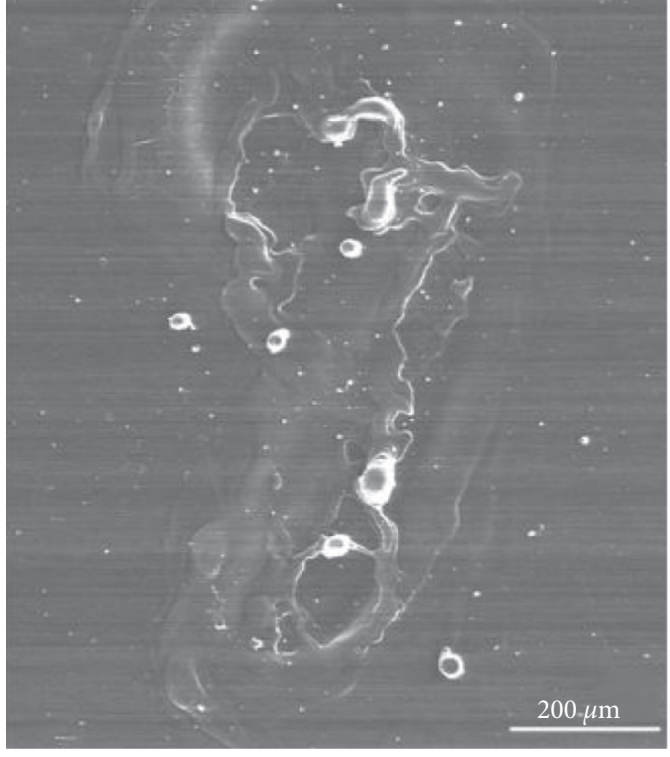

(a)
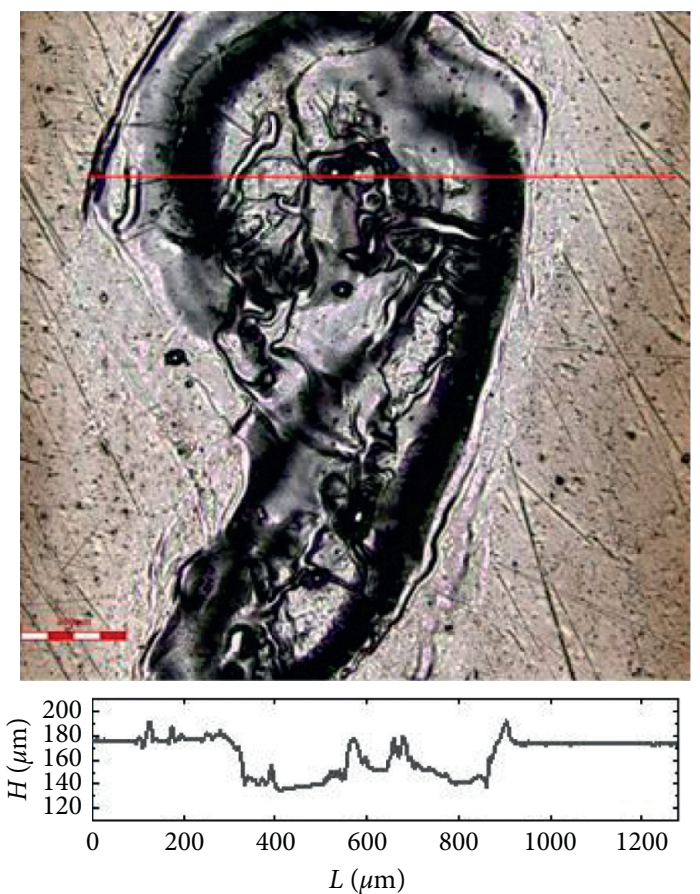

(c)

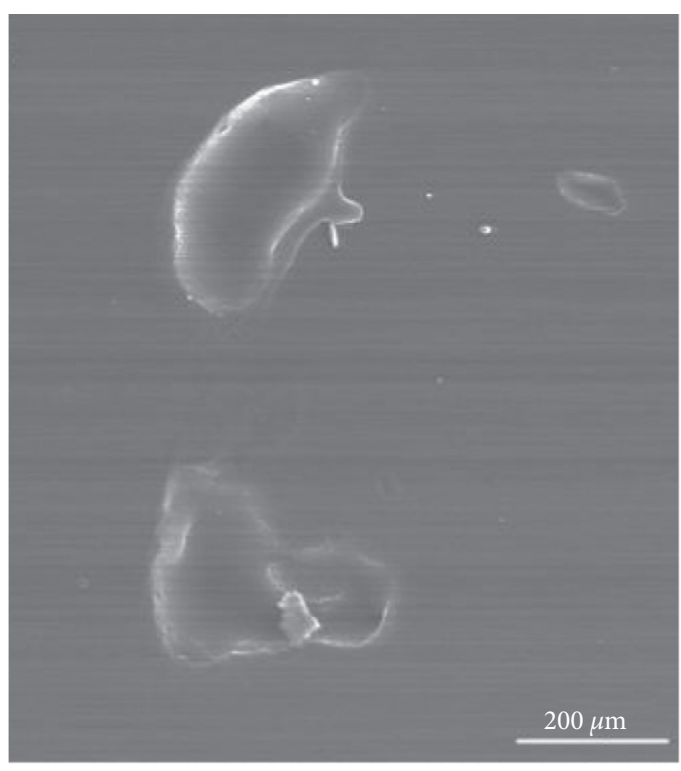

(b)
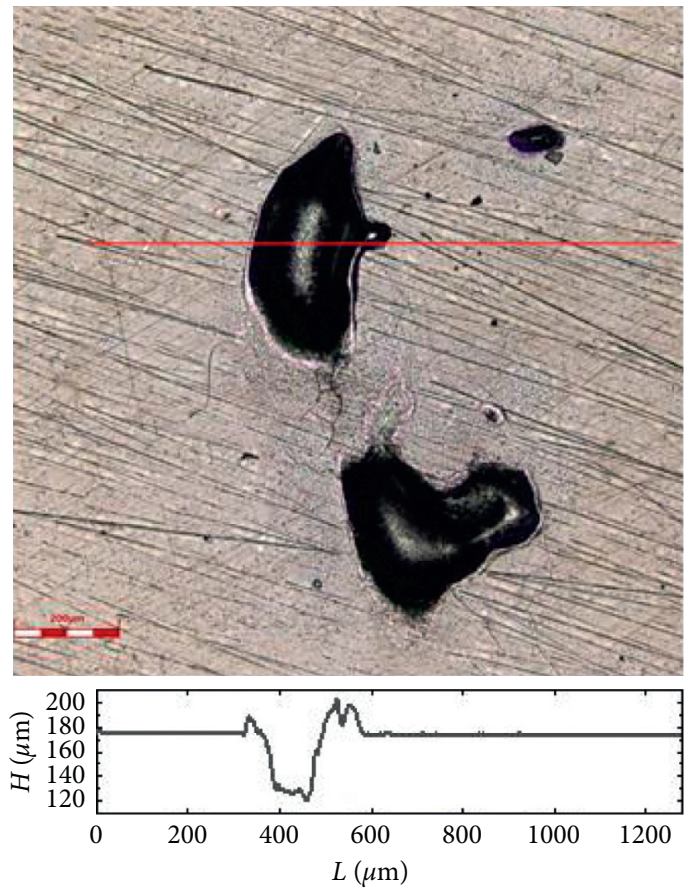

(d)

Figure 9: Microscopy images of damage traces on the titanium-alloy's witness plates using a SEM (a, b) and a CLSM (c, d). (a) Corrugated morphologies appear at a diode voltage of $166 \mathrm{kV}$ and diode current of $1.58 \mathrm{kA}$. (b) Independent, pit-like traces at a diode voltage of $303 \mathrm{kV}$ and diode current of $1.60 \mathrm{kA}$. (c) Three-dimensional morphologies of corrugated morphologies. (d) Three-dimensional morphologies of independent, pit-like traces.

alloy, so the penetration depth of electrons in SS is shallower, resulting in shallower ablative traces. In addition, unlike SS, no craters can be observed in the vicinity of corrugated morphologies after impacted by low-energy electrons (around $160 \mathrm{keV}$ ). The area where the subsurface of titanium-alloy melts is deeper than that of SS; therefore, it is quite difficult for molten droplets to break through the titanium-alloy's surface and erupt to form craters. If the electron energy is reduced $(<160 \mathrm{keV})$, craters may emerge after interaction with electrons.

Subsequently, a HPM-generation experiment on the $\mathrm{X}$-band RBWO is performed on TPG-1000 high-current electron beam accelerator. Breakdown traces are evident on the SWSs' working surfaces after the experiment, as shown in Figure 10. The breakdown traces on both sides of several SWSs show distinct symmetry after about 100 pulses 


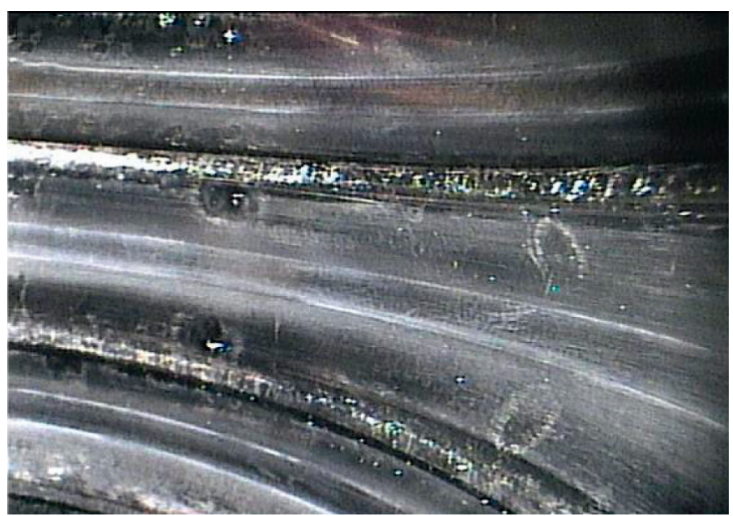

(a)

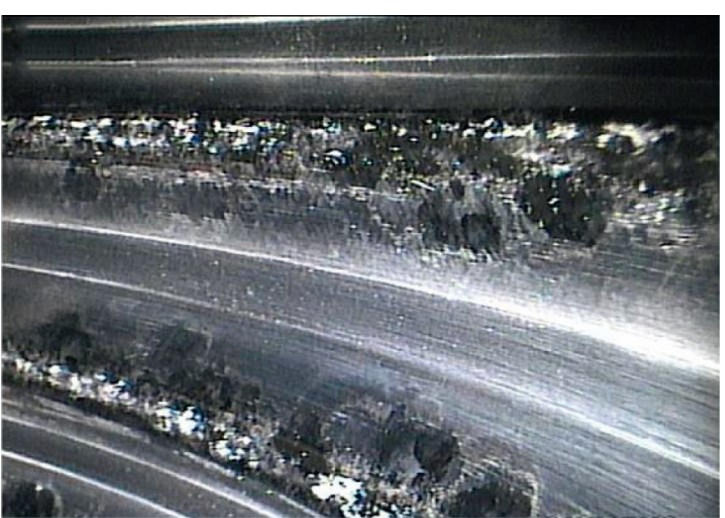

(b)

FIGURE 10: Evident breakdown traces in SWSs after the HPM-generation experiment: (a) after about 100 pulses. (b) After about 2000 pulses.

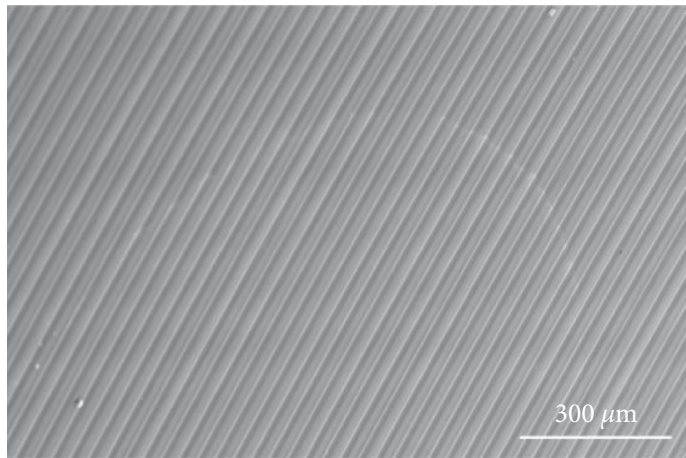

(a)

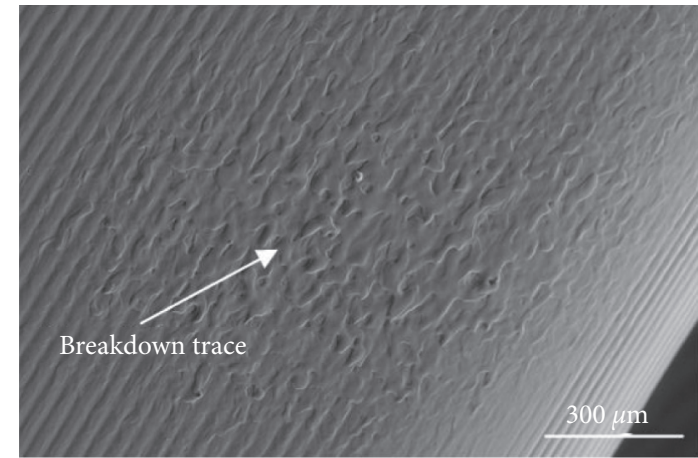

(b)

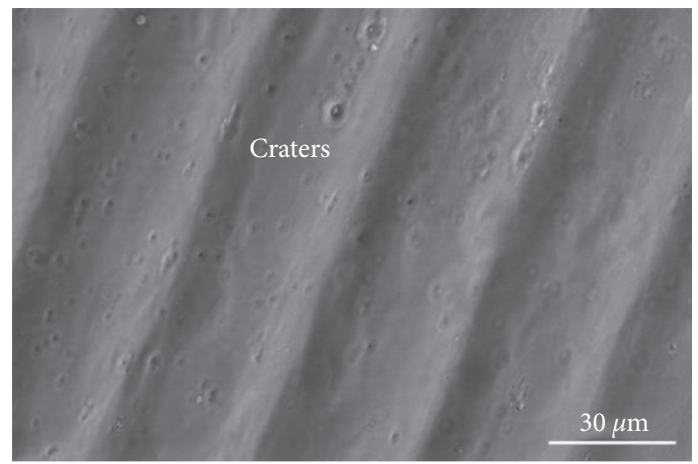

(c)

Figure 11: Typical micromorphologies of the SS SWSs: (a) before HPM experiment. (b) Corrugated morphology. (c) Craters.

(Figure 10(a)), which is in accordance with the PIC simulation and single-particle calculation, the field-induced electrons generated by some SWS ridges bombard the opposite sides and simultaneously impact on their emitting sides. As the number of pulses increases, the symmetry of breakdown traces tends to be blurred (Figure 10(b)).

Images of the breakdown traces on the working surface of left wall of the fourth SWS are obtained using a SEM and are shown in Figure 11 (SS's surface) and Figure 12 (titanium-alloy's surface). The surface of SS shows regularly spaced lines which are generated by mechanical polishing before HPM experiment (Figure 11(a)). The breakdown traces on the SS's surface entirely exhibit corrugated morphologies rather than deep pit-like traces (Figure 11(b)), and many craters emerge in the vicinity of corrugated morphologies (Figure 11(c)). These appearances are quite similar to those concerning traces left by low-energy electron bombardment. In addition, the breakdown traces on the titanium-alloy's surface exhibit shallower, wrinkle-shaped morphologies when compared to the traces from low-energy electron bombardment (around $160 \mathrm{keV}$ ). Simultaneously, a large number of craters are observed in the vicinity of wrinkle-shaped morphologies (Figure 12(c)), while no craters can be observed after direct low-energy electron 


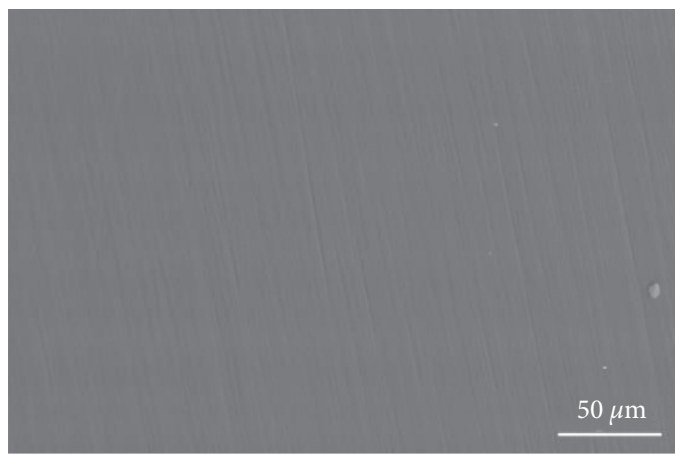

(a)

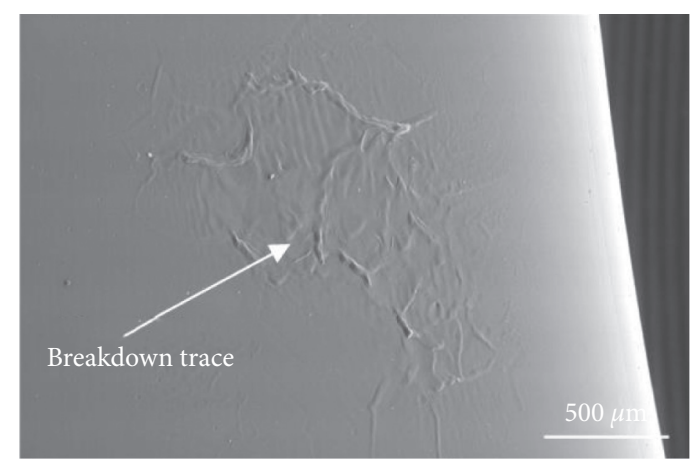

(b)

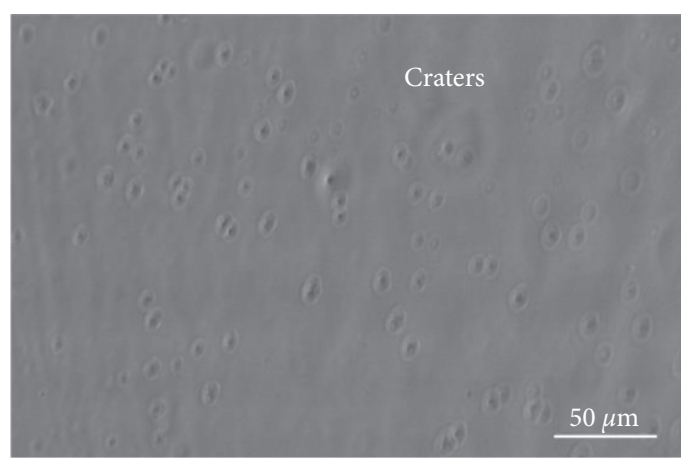

(c)

FIgURE 12: Typical micromorphologies of the titanium-alloy SWSs: (a) before HPM experiment. (b) Corrugated morphology. (c) Craters.

bombardment. These findings indicate the energy of the majority of electrons that impact on SWS surfaces, therefore, is less than $160 \mathrm{keV}$; this is in good agreement with the PIC simulation and single-particle calculation.

\section{Conclusion}

This paper presents PIC simulation, single-particle calculation, and experimental research to explore the energy range of electrons bombarding the SWS's surfaces in an $\mathrm{X}$-band RBWO. The investigation reveals that the field-induced electrons can bombard the opposite side or impact back on the emitting side, the energy of which is mainly less than $120 \mathrm{keV}$. Through the comparison of the micro appearances of the witness targets bombarded directly by electrons with various energy levels and the breakdown traces in SWSs, it is determined that the energy of electrons bombarding the surface of the structures is mainly below $160 \mathrm{keV}$, which is in accordance with the PIC simulation and numerical calculation results. In future work, we intend to explore possible suppression methods to restrain breakdown in RBWOs.

\section{Data Availability}

The data that support the findings of this study are available from the corresponding author upon reasonable request.

\section{Conflicts of Interest}

The authors declare that they have no conflicts of interest.

\section{References}

[1] A. V. Gunin, A. I. Klimov, S. D. Korovin et al., "Relativistic $\mathrm{X}$-Band BWO with 3-GW output power," IEEE Transactions on Plasma Science, vol. 26, no. 3, pp. 326-331, 1998.

[2] A. I. Klimov, I. K. Kurkan, S. D. Polevin, V. V. Rostov, and E. M. Tot'meninov, "A multigigawatt $\mathrm{X}$-band relativistic backward wave oscillator with a modulating resonant reflector," Technical Physics Letters, vol. 34, no. 3, pp. 235-237, 2008.

[3] Y. Tang, L. Meng, H. L. Li, L. Zheng, B. Wang, and F. N. Zhang, "Design of a high-efficiency dual-band coaxial relativistic backward wave oscillator with variable coupling impedance and phase velocity," Laser and Particle Beams, vol. 31, pp. 55-62, 2012.

[4] S. D. Korovin, I. K. Kurkan, S. V. Loginov, I. V. Pegel, S. D. Polevin, and S. N. Volkov, "Decimeter-band frequencytunable sources of high-power microwave pulses," Laser and Particle Beams, vol. 21, pp. 175-185, 2003.

[5] Z. Fan, Z. Song, H. Wang et al., "Direct generation of a circularly polarized TE11 mode in a relativistic backward wave oscillator by loading anisotropic medium," Journal of Physics D: Applied Physics, vol. 54, no. 7, Article ID 075206, 2021.

[6] Y. Teng, T. Z. Liang, and J. Sun, "Generation of beating wave by multi-coaxial relativistic backward wave oscillator," Laser and Particle Beams, vol. 31, pp. 703-714, 2013.

[7] X. Ge, C. Zhao, P. Zhang, F. Dang, J. Yang, and J. Zhang, “An \$S\$ -band long-pulse relativistic backward-wave oscillator with coaxial extractor," IEEE Transactions on Plasma Science, vol. 47, no. 2, pp. 1243-1248, 2019.

[8] Z. Jin, J. Zhang, J. Yang et al., "A repetitive S-band long-pulse relativistic backward-wave oscillator," Review of Scientific Instruments, vol. 82, no. 8, Article ID 084704, 2011. 
[9] D. Zhang, J. Zhang, H. Zhong, and Z. Jin, "Analysis of the mode composition of an X-band overmoded O-type Cerenkov high-power microwave oscillator," Physics of Plasmas, vol. 19, no. 10, Article ID 103102, 2012.

[10] J. Zhang, D. Zhang, Y. Fan et al., "Progress in narrowband high-power microwave sources," Physics of Plasmas, vol. 27, no. 1, Article ID 010501, 2020.

[11] R. Z. Xiao, X. W. Zhang, L. J. Zhang et al., "Efficient generation of multi-gigawatt power by a klystron-like relativistic backward wave oscillator," Laser and Particle Beams, vol. 28, no. 3, pp. 505-511, 2010.

[12] S. D. Korovin, V. V. Rostov, and E. M. Tot'meninov, “A relativistic backward wave oscillator with a modulating resonance reflector," Technical Physics Letters, vol. 31, no. 5, pp. 411-413, 2005.

[13] W. Song, C.-H. Chen, L.-G. Zhang et al., "A dual-resonant reflector in powerful relativistic backward wave oscillator," Physics of Plasmas, vol. 18, no. 6, Article ID 063105, 2011.

[14] C. S. Mayberry, B. Wroblewski, E. Schamiloglu, and C. B. Fleddermann, "Suppression of vacuum breakdown using thin-film coatings," Journal of Applied Physics, vol. 76, no. 7, pp. 4448-4450, 1994.

[15] J. Norem, Z. Insepov, and I. Konkashbaev, "Triggers for RF breakdown," Nuclear Instruments and Methods in Physics Research Section A: Accelerators, Spectrometers, Detectors and Associated Equipment, vol. 537, no. 3, pp. 510-520, 2005.

[16] M. D. Karetnikov, "An origin of RF breakdown in vacuum," Particle Accelerators, vol. 57, pp. 189-214, 1997.

[17] F. R. Schwirzke, "Vacuum breakdown on metal surfaces," IEEE Transactions on Plasma Science, vol. 19, no. 5, pp. 690-696, 1991.

[18] F. Schwirzke, M. P. Hallal Jr, and X. K. Maruyama, "Onset of breakdown and formation of cathode spots," IEEE Transactions on Plasma Science, vol. 21, no. 5, pp. 410-415, 1993.

[19] A. V. Batrakov, S. A. Onischenko, D. I. Proskurovsky, and D. J. Johnson, "Priorities of cathode and anode contaminations in triggering the short-pulsed voltage breakdown in vacuum," IEEE Transactions on Dielectrics and Electrical Insulation, vol. 13, no. 1, pp. 41-51, 2006.

[20] N. F. Kovalev, V. E. Nechaev, M. I. Petelin, and N. I. Zaitsev, "Scenario for output pulse shortening in microwave generators driven by relativistic electron beams," IEEE Transactions on Plasma Science, vol. 26, no. 3, pp. 246-251, 1998.

[21] F. Hegeler, C. Grabowski, and E. Schamiloglu, "Electron density measurements during microwave generation in a high power backward-wave oscillator," IEEE Transactions on Plasma Science, vol. 26, no. 3, pp. 275-281, 1998.

[22] S. A. Barengolts, M. Y. Kreindel, and E. A. Litvinov, "Initiation of explosive electron emission in microwave fields," IEEE Transactions on Plasma Science, vol. 26, no. 3, pp. 252-255, 1998.

[23] Y. Cao, J. Sun, Y. Zhang et al., "RF breakdown of the resonant reflector in a relativistic backward wave oscillator," IEEE Transactions on Plasma Science, vol. 46, no. 4, pp. 900-908, 2018.

[24] N. Tan, P. Wu, Y. Hua, J. Sun, W. Huang, and W. Huang, "Experimental study of destructive effects on materials with different energy electron bombardment," AIP Advances, vol. 11, no. 1, Article ID 015349, 2021.

[25] J. Wang, D. Zhang, C. Liu et al., "UNIPICcode for simulations of high power microwave devices," Physics of Plasmas, vol. 16, no. 3, Article ID 033108, 2009.

[26] R. H. Fowler and L. Nordheim, "Electron emission in intense electric fields," Proceedings of the Royal Society of London. Series A, vol. 119, pp. 173-181, 1928.
[27] H. Chen, Y. Du, W. Gai et al., "Surface-emission studies in a high-field rf gun based on measurements of field emission and Schottky-enabled photoemission," Physical Review Letters, vol. 109, no. 20, Article ID 204802, 2012.

[28] A. Grudiev, S. Calatroni, and W. Wuensch, "New local field quantity describing the high gradient limit of accelerating structures," Physical Review Special Topics-Accelerators and Beams, vol. 12, no. 10, Article ID 102001, 2009.

[29] N. d. Jonge, M. Allioux, M. Doytcheva, and M. M. Kaiser, "Characterization of the field emission properties of individual thin carbon nanotubes," Applied Physics Letters, vol. 85, pp. 1607-1609, 2004.

[30] J. X. Zou, K. M. Zhang, C. Dong, Y. Qin, S. Z. Hao, and T. Grosdidier, "Selective surface purification via crater eruption under pulsed electron beam irradiation," Applied Physics Letters, vol. 89, Article ID 041913, 2006. 